\title{
SpRoUTS (Space Robot Universal Truss System): Reversible Robotic Assembly of Deployable Truss Structures of Reconfigurable Length
}

\author{
Benjamin Jenett ${ }^{1,4}$, Daniel Cellucci ${ }^{2,4}$, Kenneth C. Cheung ${ }^{3,4}$
}

\begin{abstract}
Automatic deployment of structures has been a focus of much academic and industrial work on infrastructure applications and robotics in general. This paper presents a robotic truss assembler designed for space applications the Space Robot Universal Truss System (SpRoUTS) - that reversibly assembles a truss from a feedstock of hinged and flat-packed components, by folding the sides of each component up and locking onto the assembled structure. We describe the design and implementation of the robot and show that the assembled truss compares favorably with prior truss deployment systems.
\end{abstract}

\section{INTRODUCTION}

This work draws strongly upon two fields: the design and construction of monolithic deployable truss structures and of robots that assemble erectable truss structures from a feedstock of components.

\section{A. Space Deployables}

Deployable truss structures have often been used to address the unique structural requirements of the space environment[1], [2]. The combination of severe launch loading, volume and total mass constraints, and low (as-deployed in-service) loading requirements necessitates the use of structural systems that display packing efficiencies on the order of 100:1[3], [4]. Proposed solutions to this set of design constraints focus on the design of a monolithic device that can be packed tightly and, either by the controlled release of internally stored energy or via external mechanisms, expands to the fullly deployed length.

Mikulas[5] characterized the theoretical performance of three types of deployable trusses: tubular, longeron-based trusses, and isogrid walls. Many have explored these different types with a diverse array of solutions, including coilables[6], inflatable-rigidizables (both longeron[7] and isogrid[2]), extremely high expansion foldables[3], and skewed-compression systems[8], [9], [10].

\section{B. Robotic Assembly}

The goal of robotic assembly of large structures from modular components has seen a variety of proposed solutions. These include robots arms with many degrees of

\footnotetext{
*This work was supported by the NASA STMD ARC Center Innovation Fund and the NASA Space Technology Research Fellowship.

${ }^{1}$ Center for Bits and Atoms, Massachusetts Institute of Technology, Cambridge, MA 02139, USA be jemit. edu

${ }^{2}$ Spacecraft Lab, Cornell University, Ithaca, NY 14853, USA dwc238@cornell.edu

${ }^{3}$ National Aeronatics and Space Administration Ames Research Center, Moffett Field, CA 94035, USA kenny@nasa.gov

${ }^{4}$ Coded Structures Lab, National Aeronatics and Space Administration Ames Research Center, Moffett Field, CA 94035, USA
}

freedom relative to the built structure[11], [12], assembly with UAVs[13], robots which crawl along the built structure to place elements[14], [15], and construction platforms[16], [17].

The use of unattached robot arms involves multiple degree-of-freedom (DOF) manipulators to place elements in their correct positions. Research has focused on space applications[11], [12], [18], where they are commonly referred to as "satellite arms." A notable variation is the use of robotic aerial vehicles to perform placement, where multiple robots act in tandem to collect pieces from a source and place them in the desired locations. In either case, the separation of the assembler from the structure requires a global external positioning system in order to align the elements, adding to the complexity and limiting the scale of the final design to that of the positioning system[11], [12], [13].

Prior work that uses the built structure as a reference to place the next set of elements include robots that traverse bidirectionally geared truss members connected at nodes[15] and bricklaying robots that manipulate and place volumetric elements with integral latching interfaces[19].

The work of Galloway, et al.[16] most closely relates to the ideas proposed here. It uses an established base plane, referred to as a factory floor, to assemble each layer of the system, with a final elevator mechanism that clears the building area and enables another layer to be constructed below the previous. By connecting many of these floors in a two-dimensional array,

Most of the strategies proposed focus on designs that employ mechanical struts locking into node parts. These studies have made good progress, especially given the apparently intrinsic need for complex interlocking structures and robots that can manipulate these elements. Galloway[16], Staritz[11], and Senda[12], show multiple-DOF arms integrated into a moving super structure to enable the range and precision required to place all of the elements.

The systems proposed by Galloway[16] and Nigl[15] include procedures to perform modifications of the components of the final structure. In the former case, a flexure-based clamp to attaches struts to nodes, and in the latter bidirectional gear teeth completely cover the strut members in order to enable robot locomotion.

\section{Performance and Reversibility}

Robotic assembly systems like Galloway[16] and Nigl[15] also reference the capacity of their designs to be disassembled in addition to being assembled. This enables many useful features, including the repair of damaged sections and 
the ability to reuse material and to adapt the structure to new conditions. The addition of this capacity does not necessarily preclude high-performance, as evidence by the high modulus, ultralight reversibly assembled cellular composite materials introduced by Cheung et. al.[20].

In this work we attempt to build on these two approachesdeployable structures and robotic assembly. By assembling an erectable structure from modular components, we see an opportunity to reduce the complexity of both the assembler that produces the structure and the structural elements that compose the system. Moreover, we seek to combine the progress of these fields as well, taking the rigorous definition of design criteria central to to the study of robotic assemblers and combining it with the mature experimental and analytical testing tools of deployable structures.

\section{DESIGN CRITERIA}

This work attempts to create an effective assembler of deployable elements for space applications. As noted in Galloway[16], materials engineering and robotic design, are highly coupled. To simplify the problem, we seek to build our system around three criteria for both the assembler and the truss element: Competitive Performance, Simplicity, and Compatibility. The ways in which these criteria apply to truss elements and assembler robots are complementary.

Our objectives for the truss elements are:

- Competitive Performance that is comparable to existing structural schemes in terms of stiffness, mass efficiency, and volume efficiency.

- Simplicity in the physical design implementation of the deployable unit, with a focus on manufacturing processes that allow for mass production of the final product.

- Compatibility with the robotic assembler that will be assembling the elements together.

Our corresponding objectives for the assembler are:

- Competitive Performance in terms of the energy consumption, the number of motions, and the speed of assembly compared to other robotic assemblers.

- Simplicity in the number of degrees of freedom necessitated by the assembler to perform the motions that produce a valid structure.

- Compatibility with the truss element such that motions require the minimum of feedback.

\section{DESIGN OVERVIEW}

Fully automated robotic structure manufacturing systems have been proposed many times[15], [16], [11], [19], often employing multiple specialized robots to carry out the desired tasks such as assembly, supply, and repair. The robot we will be describing here is called "SpRoUTS". Its function is to assemble the truss beam from the packaged units by folding them into the desired shape. We plan to introduce robots that enable other functionality, such as the repair of units or position of beams to form a $3 \mathrm{~d}$ structure in subsequent paper.
The essential motions that produce the structure by folding are central to the design of the robot. Therefore, "SpRoUTS" can be thought of as a mechanical entity which implements those motions, and the truss unit as the entity which receives those motions and successfully translates them into a rigid structure. As a result, it is advantageous to describe functionality of the structure before describing the mechanisms which impart that functionality, and a video showing an animation of one assembly cycle is included in the supplementary materials.

\section{A. Unit design}

The units that comprise the truss beam are designed with simplicity in mind. The reasons for this are two-fold. The first is to ensure compatibility with existing systems of mass production such as stamping or injection molding, where many thousands of parts can be made, but where highly complex geometries such as those capable of being prototyped through $3 \mathrm{~d}$ printing will be difficult to reproduce. The second is to ensure that the motions and effectors required to configure the structure can also be made as simply as possible. As a result, the beam units were composed of HDPE, and were produced on a 3-axis mill. Producing a 2.5-d geometry that could be easily converted over to an injection-molding process.

There are two important features of the truss beam that enable the deployable and erectable behaviors. Figure 1 shows a diagram illustrating these two behaviors. The first feature is a set of living hinges, which allows the sides of the flat element to fold upward. This allows a large number of constituent elements to be stored in a configuration that conserves as much volume as possible. The second is a locking mechanism that allows the sides of the unit to attach to the base of the unit above it. This allows the structure to be connected together, enabling the desired erectable behavior. The goal of these locks is to connect one element to another, and enable the load bearing behavior of the structure to be performed by the struts of the truss rather than the hinges that enable the folding.

As a result of this design, the essential motions for the construction of one bay of the truss beam are as follows:

1) $z$ top: the top unit (or starting piece) moves to a $z$ position such that the locking mechanisms on the base of this unit are located at the proper elevation.

2) fold up: the sides of the bottom unit fold upward toward the center of the structure, such that the attachment points of the bottom unit are aligned with the locking mechanisms on the base of the top unit.

3) snap: the sides of the bottom unit overcome the internal energy of the locking mechanism, forming a mechanical connection between the sides of the bottom unit and the base of the top unit.

The bottom unit then moves in the z-direction to the vertical location of the top unit, and the whole process is repeated. The overall set of motions performed by the robot is shown in Figure 5. 

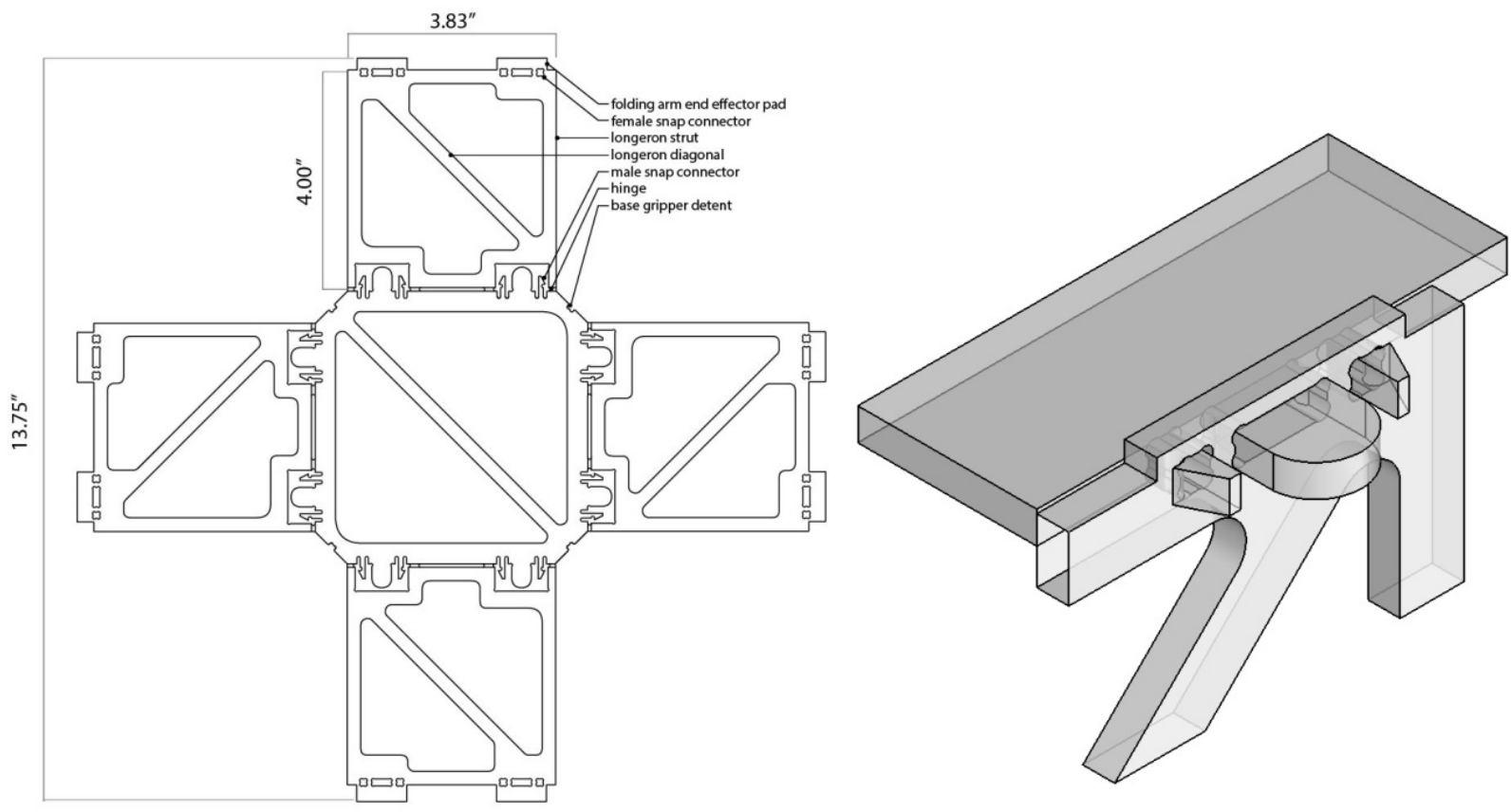

Fig. 1. A single unit of the truss, showing the deployable functionality and the locking mechanism that allows for a unit to attach to the unit above it

\section{B. Robot Design}

As a result of this physical design, our robot that constructs a truss beam from this folding and locking procedure is designed to perform two decoupled sets of motions to perform these actions. The first is the folding action, whereby a set of arms lift the sides upward and lock them into the base of the previous unit. The second is an elevator mechanism, where the robot moves the base of the finished unit to the correct vertical position to receive the arms from the next unit's folding action. The mechanisms that enable this motion are described in detail below and shown in Figure 2.

1) The Folding Arms: The folding mechanism consists of two parts, four servo-controlled arms that fold the sides upward and provide the foce necessary to mate the bottom unit with the base of the top, and four base grippers which stablize the bottom unit while the arms fold the sides.

Each arm consists of a Dynamixel MX-28T connected to a linkage with neoprene-wheel end effectors that translates the motor torque into the necessary force to mate the sides of the truss with the base plate of the previous element. Each individual end effector consists of four $1 / 2$ diameter, pressfit drive rollers. These evenly distribute pressure around the snap connection, ensuring a reliable lock.

The mechanism that enables the folding is a modified Watt's linkage, with a ground link separation that is reduced to allow a 0 -rocker motion for the input link and a $\pi$-rocker motion for the output[21].

The rest position for the mechanism is such that the floating link lies under the flattened truss unit. When the servo rotates the input link, it raises the floating link and causes the output link to rotate along with the truss side. As the truss side approaches the locked position with its predecessor, the floating link and input link approach a singularity, maximizing the mechanical advantage imparted by the linkage. The lower right drawing in Fig 3 illustrates this mechanism, including the constituent servos and the motions that the linkage produces.

In addition to the folding motion, four base-gripper mechanisms ensure that the base of the bottom unit is mechanically constrained during folding. This ensures reliable mating between the sides of the bottom unit the the base of the top during folding. The base grippers are slider-crank mechanisms, with a forked end effector which locks into a detent feature on the truss panel. The crank is a mini-size servo, which is mounted to one corner of the bottom base plate. A servo horn rotates an aluminum link, which then applies a force to the end-effector shuttle. Two parallel rails guide the shuttle with PTFE sleeve bearings. The pronged end effector also serves the purpose of disengaging an integrated tab on the panel which joins it to the unit below it in the cartridge (described in more detail in the subsection Material Feed, located below.)

2) The Base Plane Elevator: The elevator mechanisms are responsible for both stablizing the top unit as the bottom snaps into its base, and for moving the structure so that the next layer can be placed. They must therefore be able to both grip and release a unit, and also translate the structure so the next unit can mate with the locking mechanisms of the previous.

Two threaded stepper motors enable the $z$-axis translation 

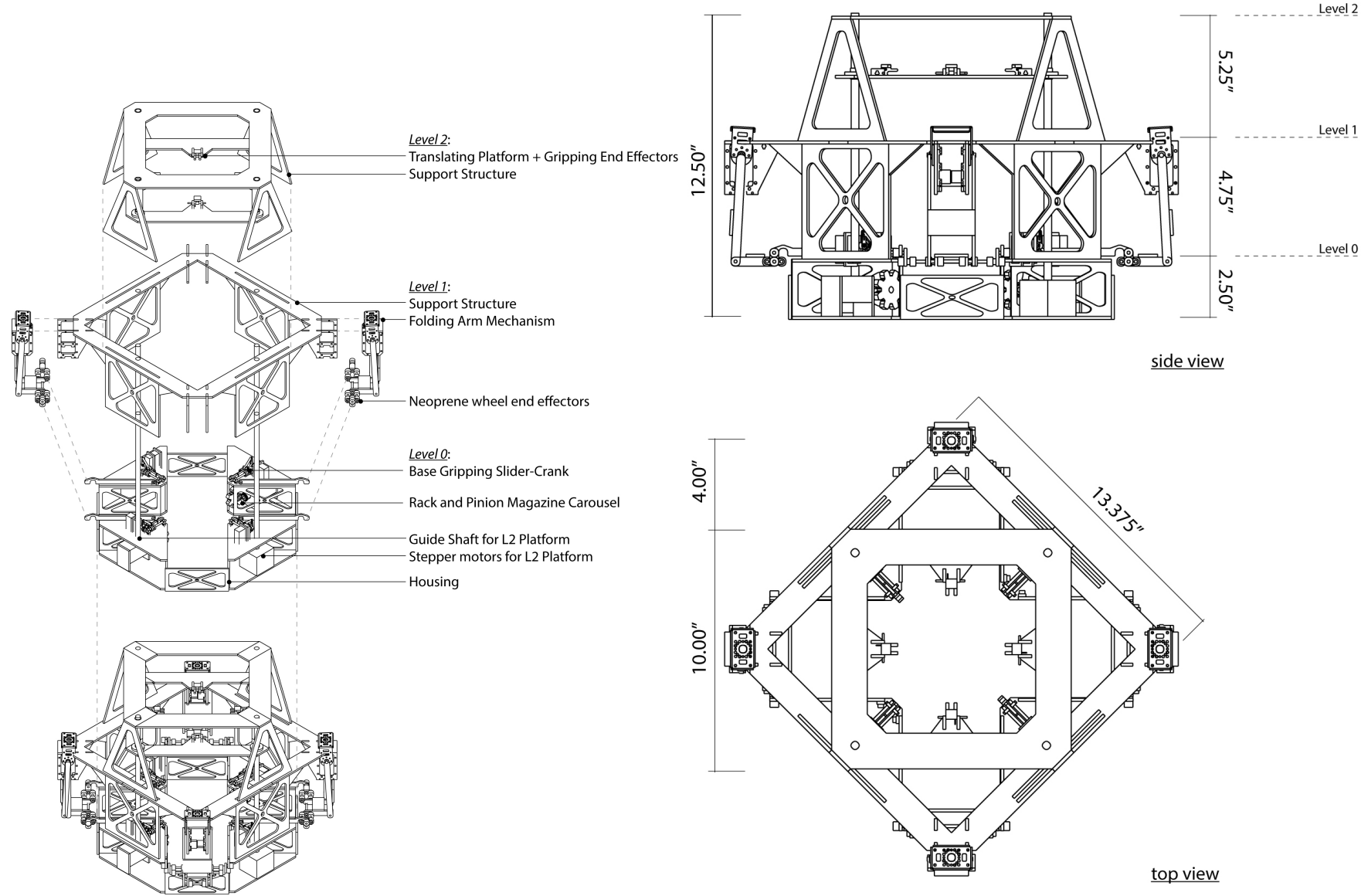

Fig. 2. Schematic view showing the various elements of the robotic assembler, as well as their overall dimensions.

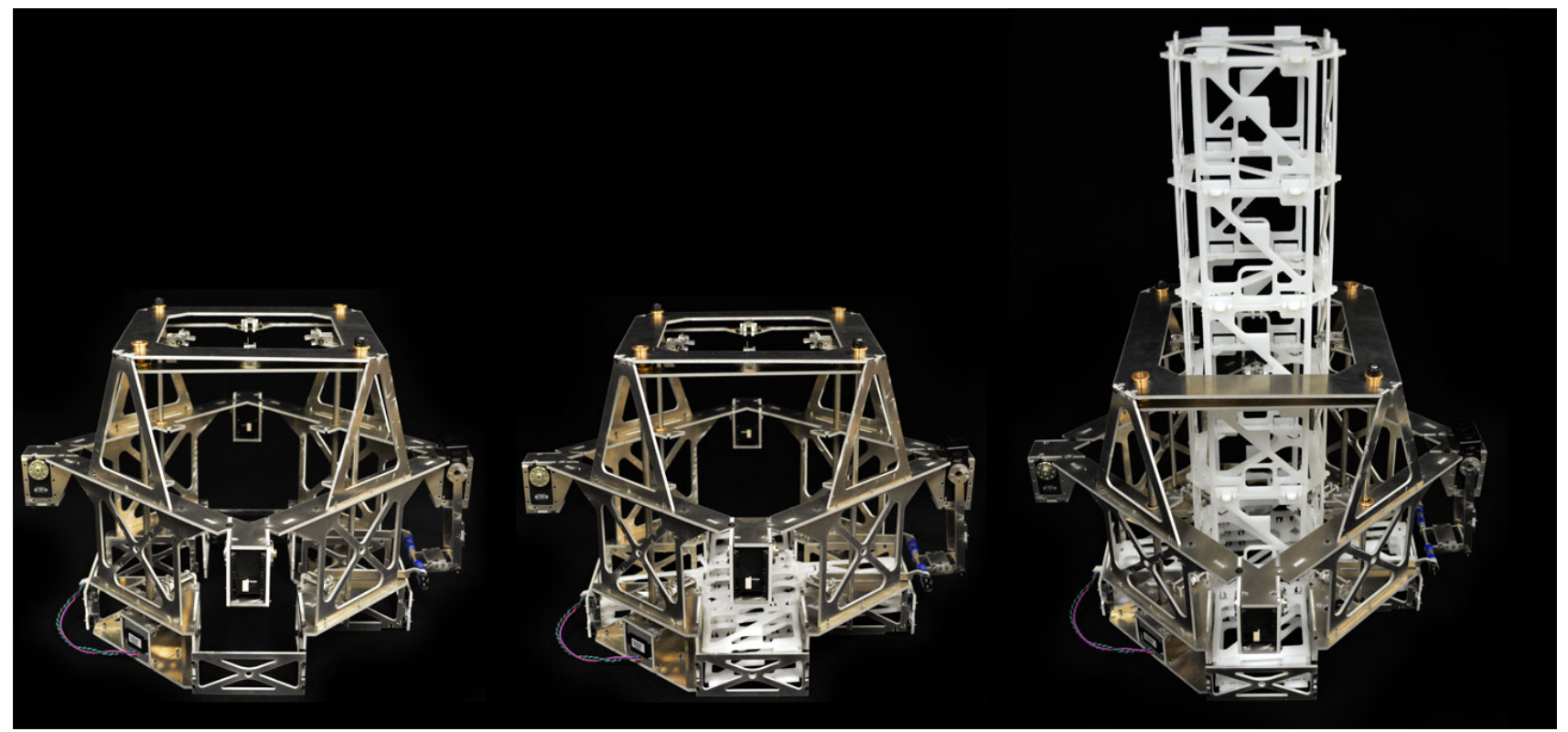

Fig. 3. A diagram showing the overall layout of the robot, including the storage cartridge for flat pack elements, and a built structure rising out of the robot. 
of the structure. These motors are located across from one another, at $0 \mathrm{deg}$ and $180 \mathrm{deg}$, with two two guide shafts located at $90 \mathrm{deg}$ and $270 \mathrm{deg}$. The shafts reduce the amount of power supply required while maintaining balanced movement of the platform.

The motors used are NEMA 17 hybrid bipolar stepping motors, each of which comes with an integrated $28 \mathrm{~cm}$ (11) threaded rod as its output shaft, turning it into a linear actuator capable of precision open-loop positioning. Each phase draws $1.7 \mathrm{~A}$ at $2.8 \mathrm{~V}$, allowing for a holding torque of $3.7 \mathrm{~kg}-\mathrm{cm}$ (51 oz-in). Therefore total power and current draw is $3.4 \mathrm{~A}$ at $2.8 \mathrm{~V}$ for holding torque of $7.4 \mathrm{~kg}-\mathrm{cm}(102$ oz-in).

The end effectors for the platform are responsible for gripping the structure to translate and stabilize it. They consist of a pairs of clips with an internal torque spring (spring constant 0.25 in-lb). This, combined with a hard stop at the clip's base, results in a one-directional gripper: when translating upward, the hard stop is engaged, forcing the truss unit to move along with the elevator. Additionally, when the next unit snaps into the base of the held unit, these grippers provide a vertical constraint that prevents the unit from moving. When the platform translates downward, the grippers are pushed out of the way by the unit, snapping back into position at the next gripping level, when the spring returns the gripper to its locked position. This is, in effect, a passive degree of freedom, which avoids additional mass, electrical supply, and programming requirements of a separate set of actuated end-effectors.

\section{Material Feed}

In addition to the manufacturing motions, an additional functionality performed by the assembler was the automated feed of additional truss units from a large magazine located below the structure. The magazine carousel is a rack-andpinion mechanism, where the stacked part magazine is the rack, and a custom gear driven by a stepper motor is the pinion. Turning the gear of the rack lifts the entire cartridge of parts, moving the next bottom unit into position to be grabbed the base-gripper.

In order to enable this feed functionality, we performed two major modifications to the system, one to the folding arms of the robot, and one to the material itself. The rest location for the folder arms is such that it interferes with the vertical motion of the next part moving through the feed. As a result, we added a latching mechanism that allows the folding arm servo to move the end-effector out of the way during the feeding process. Once the next unit is in position, the servo moves the end-effector back into position and the structure can fold.

In addition, the size of the end-effector rollers means that there must be a minimum clearance between the bottom unit and the next unit in the cartridge. For this prototype, we accomplished this functionality by adding a small tab to each of the units so that inside the cartridge there is a minimum separation vertical between them. The base-gripper is also responsible for disengaging this tab so that the elevator can translate the bottom unit without also bringing the rest of the units in the cartridge with it. Future iterations can minimize the size of this separation by instead using base-grippers that have limited vertical motion in addition to horizontal, lifting the bottom unit up from the cartridge and providing the necessary clearance for the folding arms.

\section{DISCUSSION}

One of the unique difficulties of comparing performance between the various proposed solutions for space structures is finding a common set of metrics that can compare the efficacy of two approaches regardless of material selection or . Mikulas et al. [4] addresses this problem with a set of performance and packaging metrics, the most critical of which is assigned the label $\Sigma$. This parameter, given a measured stiffness $S=E I$, material modulus $E$, an overall mass $M$, an overall length $L$, a density $\rho$ and a diameter of $D$ is

$$
\frac{S}{m}=\frac{1}{8} \frac{E}{\rho \Sigma} D^{2}
$$

The value $\Sigma$ is proposed as a measure for what is described in the publication as a "parasitic mass", which includes ancillary mass not fully contributing to the stiffness of the overall structure but instead devoted to the logistics of deployment. However, this can also be thought of a material-independent measure of the ability of the constructed truss to achieve the theoretical maximum stiffness for the assembly method and geometry chosen[8]. This second interepretation allows for the comparison of a wide variety of proposed schemes across a wide range of feature scales. This includes a variety of deployable schemes, which are calculated by Mikulas et al. [4], as well as other erectable schemes described in [22] and [23].

Figure 4 shows the proposed scheme compared to other deployable schemes. With a calculated value of $\Sigma=20$, the proposed system sits on the low side of performance, but within the range of previously proposed schemes. This is compelling in part because the structure measured here was not optimized in any way for mass, instead being constrained by the manufacturing process used to produce the part and a reasonable scale and resolution for an initial protype robot. Future iterations will explore optimizations of the unit design to minimize the value of $\Sigma$, and then modify the existing folding paradigm to accomodate these optimized units.

\section{ACKNOWLEDGMENTS}

We would like to acknowledge the generous support of the NASA Space Technology Research Fellowship, the ARC SpaceShop facility, and the MIT Center for Bits and Atoms.

\section{REFERENCES}

[1] J. W. Umland and H. Eisen, "Srtm on-orbit structural dynamics," AIAA, vol. 1588, pp. 18-21, 2001.

[2] J. K. Lin, G. S. Agnes, R. D. Abelson, C. E. Tucker, J. J. Watson, and T. W. Jones, "Ultra lightweight isogrid boom space experiment (ultraboom) systems design," in 46th AIAA/ASME/ASCE/AHS/ASC Structures, Structural Dynamics, and Materials Conference, 2005, pp. $1-12$. 


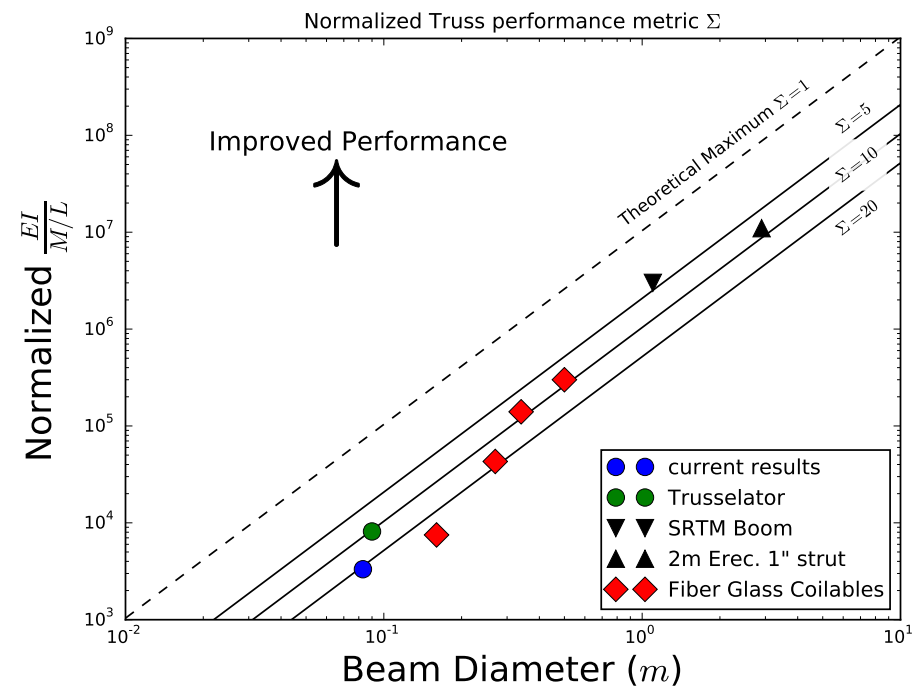

Fig. 4. Normalized structural performance, showing scale- and material- independent parameter $\Sigma$

ZTOP FOLDUP SNAP FOLD DOWN
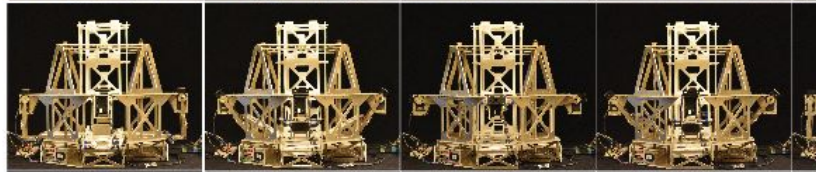

ZDOWN

Z воттом

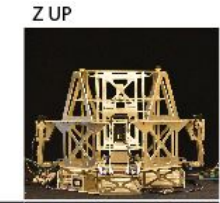

Tratestent

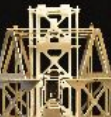

A

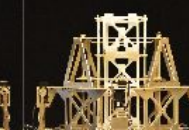

b彭 dis $=1$ I Non 40 -

to: 13

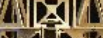
(1) 101010 Nind and

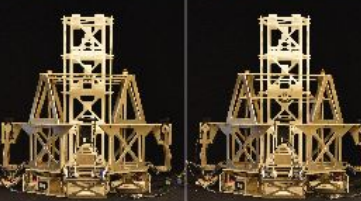
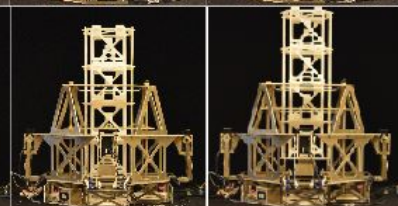

THREE
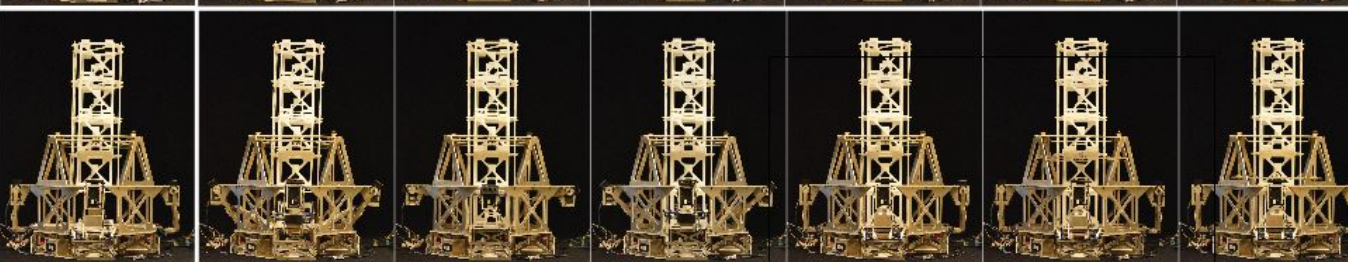

原

FOUR
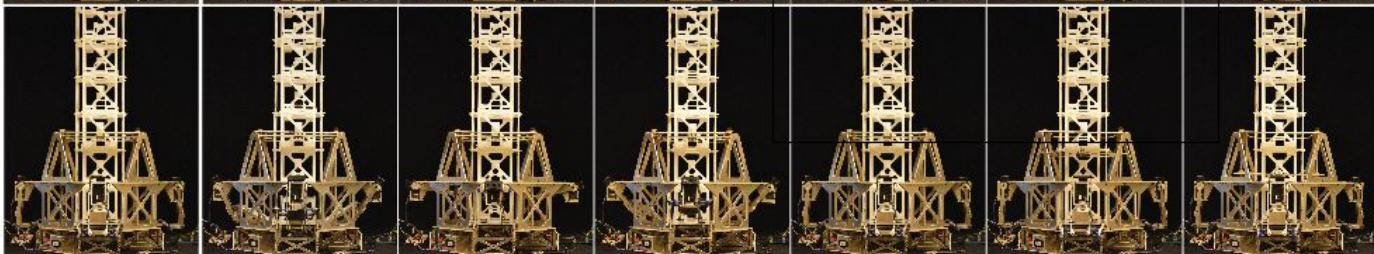

tos

|खे]

ADAII
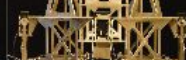

FIVE

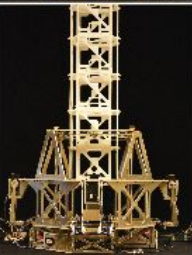

Fig. 5. The full sequence of motions to produce a six-bay truss beam using the SpRoUTS assembler 
[3] D. J. B. Warren, Peter A. and M. Silver, "Experimental characterization of an extremely high expansion deployable gossamer structure," in 48th AIAA/ASME/ASCE/AHS/ASC Structures, Structural Dynamics, and Materials Conference, 2007.

[4] M. M. Mikulas, T. J. Collins, W. Doggett, J. Dorsey, and J. Watson, "Truss performance and packaging metrics," in SPACE TECH. \& APPLIC. INT. FORUM-STAIF 2006: 10th Conf Thermophys Applic Microgravity; 23rd Symp Space Nucl Pwr \& Propulsion; 4th Conf Human/Robotic Tech \& Nat'l Vision for Space Explor;; 4th Symp Space Coloniz.; 3rd Symp on New Frontiers \& Future Concepts, vol. 813, no. 1. AIP Publishing, 2006, pp. 1000-1009.

[5] M. M. Mikulas, "Structural efficiency of long lightly loaded truss and isogrid columns for space applications," 1978.

[6] D. M. Murphy, M. E. McEachen, B. D. Macy, and J. Gaspar, "Demonstration of a 20-m solar sail system," AIAA Paper, vol. 2126, p. 2005, 2005.

[7] K. Guidanean and D. Lichodziejewski, "An inflatable rigidizable truss structure based on new sub-tg polyurethane composites," in 43rd AIAA SDM Conference Proceedings (AIAA-02-1593), 2002.

[8] M. A. Brown, "A deployable mast for solar sails in the range of 1001000m," Advances in Space Research, vol. 48, no. 11, pp. 1747-1753, 2011.

[9] M. Brown, F. Tasker, and G. Kirby, "A deployable truss beam for long or lightly loaded space applications," in 10th Gossamer Forum, 2009.

[10] J. M. Mejia-Ariza, T. W. Murphey, and H.-P. Dumm, "Deployable trusses based on large rotation flexure hinges," Journal of Spacecraft and Rockets, vol. 47, no. 6, pp. 1053-1062, 2010.

[11] P. J. Staritz, S. Skaff, C. Urmson, and W. Whittaker, "Skyworker: a robot for assembly, inspection and maintenance of large scale orbital facilities," in Robotics and Automation, 2001. Proceedings 2001 ICRA. IEEE International Conference on, vol. 4. IEEE, 2001, pp. 41804185.

[12] K. Senda, Y. Murotsu, A. Mitsuya, H. Adachi, Shin', I. Ito, J. Shitakubo, and T. Matsumoto, "Hardware experiments of a truss assembly by an autonomous space learning robot," Journal of spacecraft and rockets, vol. 39, no. 2, pp. 267-273, 2002.

[13] Q. Lindsey, D. Mellinger, and V. Kumar, "Construction with quadrotor teams," Autonomous Robots, vol. 33, no. 3, pp. 323-336, 2012.

[14] D. Hjelle and H. Lipson, "A robotically reconfigurable truss," in Reconfigurable Mechanisms and Robots, 2009. ReMAR 2009. ASME/IFToMM International Conference on. IEEE, 2009, pp. 73-78.

[15] F. Nigl, S. Li, J. Blum, and H. Lipson, "Autonomous truss reconfiguration and manipulation," 2013.

[16] K. C. Galloway, R. Jois, and M. Yim, "Factory floor: A robotically reconfigurable construction platform," in Robotics and Automation (ICRA), 2010 IEEE International Conference on. IEEE, 2010, pp. 2467-2472.

[17] R. Hoyt, J. Cushing, J. Slostad, and T. Unlimited, "Spiderfab: Process for on-orbit construction of kilometer-scale apertures," Final Report of the NASA NNX12AR13G Contract, 2013.

[18] E. Komendera, J. T. Dorsey, W. R. Doggett, and N. Correll, "Truss assembly and welding by intelligent precision jigging robots," 2014.

[19] Y. Terada and S. Murata, "Automatic assembly system for a large-scale modular structure-hardware design of module and assembler robot," in Intelligent Robots and Systems, 2004.(IROS 2004). Proceedings. 2004 IEEE/RSJ International Conference on, vol. 3. IEEE, 2004, pp. 2349-2355.

[20] K. C. Cheung and N. Gershenfeld, "Reversibly assembled cellular composite materials," science, vol. 341, no. 6151, pp. 1219-1221, 2013.

[21] J. M. McCarthy and G. S. Soh, Geometric design of linkages. Springer, 2010, vol. 11.

[22] R. P. Hoyt, J. I. Cushing, G. J. Jimmerson, J. S. Luise, and J. T. Slostad, "Trusselator: On-orbit fabrication of high-performance composite truss structures," 2014.

[23] T. J. Collins and W. Fichter, "Support trusses for large precision segmented reflectors: Preliminary design and analysis," in 1989 Orlando Symposium. International Society for Optics and Photonics, 1989, pp. $453-469$. 